\title{
Managing Incomplete Preference Relations in Decision Making: A Review and Future Trends ${ }^{\text {tr }}$
}

\author{
R. Ureña ${ }^{a}$, F. Chiclana ${ }^{b}$, J.A. Morente ${ }^{a}$, E. Herrera-Viedma ${ }^{a}$ \\ ${ }^{a}$ Department of Computer Science and A.I, University of Granada, 18071 Granada Spain \\ ${ }^{b}$ Centre for Computational Intelligence, Faculty of Technology, De Montfort University, Leicester, UK
}

\begin{abstract}
In decision making, situations where all experts are able to efficiently express their preferences over all the available options are the exception rather than the rule. Indeed, the above scenario requires all experts to possess a precise or sufficient level of knowledge of the whole problem to tackle, including the ability to discriminate the degree up to which some options are better than others. These assumptions can be seen unrealistic in many decision making situations, especially those involving a large number of alternatives to choose from and/or conflicting and dynamic sources of information. Some methodologies widely adopted in these situations are to discard or to rate more negatively those experts that provide preferences with missing values. However, incomplete information is not equivalent to low quality information, and consequently these methodologies could lead to biased or even bad solutions since useful information might not being taken properly into account in the decision process. Therefore, alternative approaches to manage incomplete preference relations that estimates the missing information in decision making are desirable and possible. This paper presents and analyses methods and processes developed on this area towards the estimation of missing preferences in decision making, and highlights some areas for future research.

Keywords: Group decision making, Uncertainty, Incomplete information, Fuzzy preferences, Consistency
\end{abstract}

\section{Introduction}

Group decision making (GDM) consists of multiple individuals interacting to choose the best option between all the available ones. Each decision maker (DM) or expert may have his/her own opinions and background and, although they might share a common interest in achieving

\footnotetext{
${ }^{\text {th }}$ Cite as: Raquel Ureña, Francisco Chiclana, Juan Antonio Morente, Enrique Herrera-Viedma: 'Managing Incomplete Preference Relations in Decision Making: A Review and Future Trends.' Information Sciences. In press, 2015. doi: 10.1016/j.ins.2014.12.061

Email addresses: raquel@decsai.ugr.es (R. Ureña), chiclana@dmu.ac.uk (F. Chiclana), jamoren@decsai.ugr.es (J.A. Morente), viedma@decsai.ugr.es (E. Herrera-Viedma)
} 
agreement on selecting the most suitable option, it is expected that they would approach the problem in different ways.

The majority of GDM problems comprise the following phases depicted in Figure 1 [36]: (1) definition of the problem; (2) analysis of the problem; (3) identification of a set of alternatives; (4) identification of the set of criteria and panel of experts; and (5) application of a selection process to derive the solution to the problem.

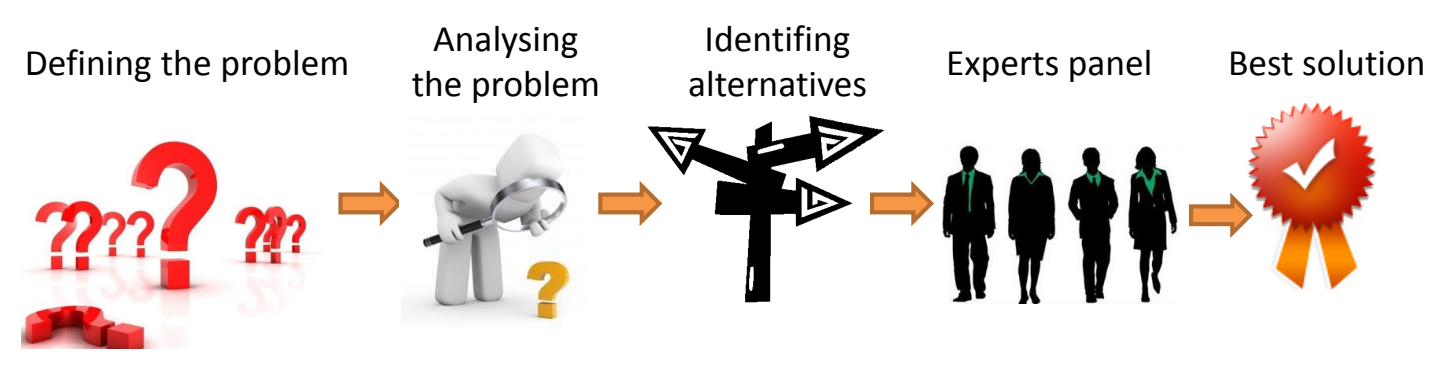

Figure 1: GDM problem resolution steps.

In GDM systems experts have to express their preferences by means of a set of evaluations over a set of alternatives. To that aim different preference representation formats are available [27]. However, it is common that an expert might not possess a precise or sufficient level of knowledge of part of the problem and, as a consequence, he/she might not provide all the information that is required $[2,14,24,44]$. Actually, situations where all experts are able to efficiently express their preferences over all the available options might be considered the exception rather than the rule. Indeed, the above scenario requires all experts to possess a precise or sufficient level of knowledge of the whole problem to tackle, including the ability to discriminate the degree up to which some options are better than others. These assumptions can be seen as unrealistic in many decision making situations, especially those involving a large number of alternatives to choose from and/or conflicting and dynamic sources of information. Indeed, a study by Deparis et al. [22] corroborates empirically the following hypothesis: "increasing the intensity of conflict in a multicriteria comparison increases the likelihood that DMs consider two alternatives as incomparable," and therefore leading to the expression of incomplete preferences. Their results indicate that a large attribute spread increases the frequency of incomparability statements when allowed, otherwise an increase of indifference statements happens. Therefore, it becomes necessary to develop decision models to address the presence 
of incomplete information, i.e. information with missing data.

Different approaches have been developed to deal with incomplete information modelled using different representation formats, which can be broadly classified into three main groups:

(i) methods that directly discard the incomplete information and process only pieces of complete information [52];

(ii) methods that penalise or rate negatively the experts who provide incomplete preferences [24]; and

(iii) methods that estimate the missing preference values using the provided ones [39, 40].

The first two groups of methods are based on the assumption that a good solution to a decision making problem cannot be achieved from incomplete information, or that the solution would not be as good as the one that would derive using complete information. However, empirical evidence suggests that the incomplete relation derived from the random deletion of as much as $50 \%$ of the elements of a complete pairwise preference relation provides good results without compromising accuracy [14]. Therefore, these two groups of methods eliminate or undervalue useful information in the data provided, which could lead to serious biases [43]. Indeed, incomplete information is not equivalent to low quality information, and consequently imposing penalties in the decision making processes to experts providing incomplete information could lead to misleading solution, specially when the incomplete information is consistent and the complete information is not. Thus, alternative approaches to manage incomplete information in decision making are desirable. One of these approaches is based on the selection of an appropriate methodology to 'build' the matrix, and/or to assign importance values to experts based not on the amount of information provided but on how consistent the information provided is.

Some of the existing methods that estimate missing preference values in GDM use the information provided by the rest of experts together with aggregation procedures [44]. The main drawback for this approach is that it requires several experts to estimate the missing values of a particular one, which in conjunction with notable difference between the experts preferences could lead to the estimation of information not naturally compatible with the rest of the expert's information. An alternative approach here is to use methods to estimate an expert's missing values using just his/her own assessments and consistency criteria to avoid incompatibility. This has been a tool extensively applied in decision making contexts under preference relations $[1-5,25,29,39,40,46,48,74]$. An extreme case of incomplete preferences happens when one or more experts in the group do not provide any preference information on at least one of the feasible alternatives. This situations are called in literature total ignorance 
or simply ignorance situations, and several approaches to deal with them have been presented in [4].

This paper presents a review of the foundations and developments in estimating missing preferences in decision making with the following different kinds of preference relations used as the preference representation format: additive, multiplicative, intuitionistic, interval and linguistic preference relations. A comprehensive analysis of the most recent developed applications in the specialised literature is presented. Finally, some of the current trends and potential future research lines of enquiry on this research topic are also outlined.

The remainder of the paper is set out as follows: In Section 2 the principal types of preference relations used in decision making are reviewed, including a description on the characterisation of their consistency. The main strategies developed to tackle the presence of incomplete preferences for the different types of preference relations will be presented in Section 3. Section 4 focuses on those cases that are being called as ignorance situations in GDM. A discussion on the current trends and future work in this research area is covered in Section 5. In Section 6 conclusions are drawn.

\section{Preference Relations in Decision Making}

In any decision making problem, once the set of feasible alternatives $(X)$ is identified, experts are called to express their opinions or preferences on such set. Different preference elicitation methods were compared in [52], concluding that pairwise comparison methods are more accurate than non-pairwise methods. A comparison of two alternatives of $X$ by an expert can lead to the preference of one alternative to the other or to a state of indifference between them. Obviously, there is the possibility of an expert being unable to compare them.

Two main mathematical models based on the concept of preference relation can be used in this context. In the first one, a preference relation is defined for each one of the above three possible preference states (preference, indifference, incomparability) [26], which is usually referred to as a preference structure on the set of alternatives [60]. The second one integrates the three possible preference states into a single preference relation [8]. For this second type of mathematical model $\mathrm{Xu}$ has carried in [86] a comprehensive review of the different types of preference relations in the literature among with some of their main properties. In this paper, we also focus on this second one.

Formally, a preference relation is defined as follows:

Definition 1 (Preference Relation (PR) [54]). A preference relation $R$ is a binary relation defined on the set $X$ that is characterised by a function $\mu_{p}: X \times X \rightarrow D$, where $D$ is the 
domain of representation of preference degrees provided by the decision maker.

When cardinality of $X$ is small, $R$ may be conveniently represented by an $n \times n$ matrix $R=\left(r_{i j}\right)$, with $r_{i j}=\mu_{p}\left(x_{i}, x_{j}\right)$ being interpreted as the degree or intensity of preference of alternative $x_{i}$ over $x_{j}$. The elements of $R$ can be of a numeric or linguistic nature, i.e., could represent numeric or linguistic preferences, respectively.

\subsection{Numeric Preferences}

The main types of numeric preference relations used in decision making are: crisp preference relations, additive preference relations, multiplicative preference relations, interval-valued preference relations and intuitionistic preference relations. In the following subsections we analyse each one of these options.

\subsubsection{Crisp Preference Relation}

When an expert is able to compare two alternatives the following broad outcomes are possible: (i) one alternative is preferred $(\succ)$ to another; or (ii) the two alternatives are indifferent $(\sim)$. Using a numerical representation of preferences, any ordered pair of alternatives $\left(x_{i}, x_{j}\right) \in X \times X$ can be associated a number from the set $D=\left\{0, \frac{1}{2}, 1\right\}$ as follows [26]:

$$
\begin{array}{rll}
r_{i j}=1 & \Leftrightarrow & x_{i} \succ x_{j} \\
r_{i j}=0 & \Leftrightarrow & x_{j} \succ x_{i} \\
r_{i j}=0.5 & \Leftrightarrow & x_{j} \sim x_{i}
\end{array}
$$

The following 'reciprocity' property is always assumed to avoid 'inconsistent' situations where

an expert could prefer two alternatives at the same time: when $r_{i j}=\frac{1}{2}$ it is also $r_{j i}=\frac{1}{2}$; and when $r_{i j}=1$ then $r_{j i}=0$.

\subsubsection{Additive Preference Relation}

The introduction of the concept of fuzzy set as an extension of the classical concept of set when applied to a binary relation leads to the concept of a fuzzy or $[0,1]$-valued preference relation, $P=\left(p_{i j}\right)[8]$, referred to as additive preference relation (APR) in this paper:

Definition 2 (Additive Preference Relation (APR) [54]). An APR $P$ on a finite set of alternatives $X$ is characterised by a membership function

$$
\mu_{P}: X \times X \longrightarrow[0,1], \mu_{P}\left(x_{i}, x_{j}\right)=p_{i j},
$$


verifying

$$
p_{i j}+p_{j i}=1 \forall i, j \in\{1, \ldots, n\} .
$$

The following interpretation is assumed:

- $p_{i j}>0.5$ indicates that the expert prefers the alternative $x_{i}$ to the alternative $x_{j}$, with $p_{i j}=1$ being the maximum degree of preference for $x_{i}$ over $x_{j}$;

- $p_{i j}=0.5$ represents indifference between $x_{i}$ and $x_{j}$.

An APR can be seen as a particular case of a (weakly) complete fuzzy preference relation [27], i.e. a fuzzy preference relation satisfying $p_{i j}+p_{j i} \geq 1 \forall i, j$.

\subsubsection{Multiplicative Preference Relation}

The measuring of the intensity of preferences can be done using a ratio scale instead, with the most widely ratio scale used being the interval $D=[1 / 9,9][61]$.

Definition 3 (Multiplicative Preference Relation (MPR)). A MPR A on a finite set of alternatives $X$ is characterised by a membership function

$$
\mu_{A}: X \times X \longrightarrow[1 / 9,9], \mu_{A}\left(x_{i}, x_{j}\right)=a_{i j}
$$

verifying

$$
a_{i j} \cdot a_{j i}=1 \forall i, j \in\{1, \ldots, n\}
$$

The following interpretation is assumed: $x_{i}$ is $a_{i j}$ times as good as $x_{j}$, and in particular:

- $a_{i j}=1$ indicates indifference between $x_{i}$ and $x_{j}$;

- $a_{i j}=9$ indicates that $x_{i}$ is absolutely preferred to $x_{j}$;

In [16], it was proved that multiplicative and additive preference relations are isomorphic:

Proposition 1. Suppose that we have a set of alternatives, $X=\left\{x_{1}, \ldots, x_{n}\right\}$, and associated with it a MPR $A=\left(a_{i j}\right)$, with $a_{i j} \in[1 / 9,9]$ and $a_{i j} \cdot a_{j i}=1, \forall i, j$. Then the corresponding $A P R, P=\left(p_{i j}\right)$, associated to $A$, with $p_{i j} \in[0,1]$ and $p_{i j}+p_{j i}=1, \forall i, j$, is given as follows:

$$
p_{i j}=f\left(a_{i j}\right)=\frac{1}{2}\left(1+\log _{9} a_{i j}\right) .
$$

The above transformation function is bijective and, therefore, allows to transpose concepts that have been defined for APRs to MPRs, and vice-versa. 


\subsubsection{Interval-Valued Preference Relation}

Membership functions of fuzzy sets are subject to uncertainty arising from various sources [51]. To reflect that Klir and Folger described blurring a fuzzy set to form an interval-valued fuzzy set [45]:

Definition 4 (Interval-Valued Fuzzy Set (IVFS)). Let INT $([0,1])$ be the set of all closed subintervals of $[0,1]$ and $X$ be a universe of discourse. An interval-valued fuzzy set (IVFS) $\widetilde{A}$ on $X$ is characterised by a membership function $\mu_{\widetilde{A}}: X \rightarrow \operatorname{INT}([0,1])$. An IVFS $\widetilde{A}$ on $X$ can be expressed as follows:

$$
A=\left\{\left(x, \mu_{\widetilde{A}}(x)\right) ; \mu_{\widetilde{A}}(x) \in I N T([0,1]) \forall x \in X\right\} .
$$

The application of the concept of IVFS to an APR leads to the concept of interval-valued APR (IVPR), i.e. a preference relation with domain of representation of preference degrees is the set of all closed subintervals of $[0,1], D=\operatorname{INT}([0,1])$.

Definition 5 (Interval-Valued Additive Preference Relation (IVPR)). An interval-valued additive preference relation (IVPR) [80] $\widetilde{P}$ on a finite set of alternatives $X=\left\{x_{1}, \ldots, x_{n}\right\}$ is characterised by a membership function $\mu_{\widetilde{P}}: X \times X \longrightarrow I N T([0,1])$, with $\mu_{\widetilde{P}}\left(x_{i}, x_{j}\right)=\widetilde{p}_{i j}=$ $\left[p_{i j}^{-}, p_{i j}^{+}\right]$, verifying

$$
\forall i, j \in\{1, \ldots, n\}: \widetilde{p}_{j i}=1-\widetilde{p}_{i j}
$$

The above definition of IVPR can be expressed in terms of the lower and upper bound of the interval-valued preference values as follows:

$$
\forall i, j=1,2, \ldots n: p_{i j}^{-}+p_{j i}^{+}=p_{i j}^{+}+p_{j i}^{-}=1
$$

\subsubsection{Intuitionistic Preference Relation}

The concept of an intuitionistic fuzzy set (IFS) was introduced by Atanassov [7]:

Definition 6 (Intuitionistic Fuzzy Set (IFS)). An intuitionistic fuzzy set (IFS) A over a universe of discourse $X$ is represented as $A=\left\{\left(x,\left\langle\mu_{A}(x), \nu_{A}(x)\right\rangle\right) \mid x \in X\right\}$ where $\mu_{A}: X \rightarrow$ $[0,1], \nu_{A}: X \rightarrow[0,1]$ and $0 \leq \mu_{A}(x)+\nu_{A}(x) \leq 1 \quad \forall x \in X$. For each $x \in X$, the numbers $\mu_{A}(x)$ and $\nu_{A}(x)$ are known as the degree of membership and degree of non-membership of $x$ to $A$, respectively.

An IFS becomes a FS when $\mu_{A}(x)=1-\nu_{A}(x) \forall x \in X$. However, when there exists at least a value $x \in X$ such that $\mu_{A}(x)<1-\nu_{A}(x)$, an extra parameter has to be taken into account 
when working with IFSs: the hesitancy degree, $\tau_{A}(x)=1-\mu_{A}(x)-\nu_{A}(x)$, that represents the amount of lacking information in determining the membership of $x$ to $A$. If the hesitation degree is zero, the reciprocal relationship between membership and non-membership makes the latter one unnecessary in the formulation as it can be derived from the former.

In [64], Szmidt and Kacprzyk defined the intuitionistic fuzzy preference relation (IFPR) as a generalisation of the concept of APR.

Definition 7 (Intuitionistic Fuzzy Preference Relation (IFPR)). An intuitionistic fuzzy preference relation (IFPR) B [87] on a finite set of alternatives $X$ is characterised by a membership function

$$
\mu_{B}: X \times X \rightarrow[0,1]
$$

and a non-membership function

$$
\nu_{B}: X \times X \rightarrow[0,1]
$$

such that

$$
0 \leq \mu_{B}\left(x_{i}, x_{j}\right)+\nu_{B}\left(x_{i}, x_{j}\right) \leq 1 \quad \forall\left(x_{i}, x_{j}\right) \in X \times X .
$$

An IFPR can be conveniently represented by a matrix $B=\left(b_{i j}\right)$ with $b_{i j}=\left(\mu_{i j}, \nu_{i j}\right) \forall i, j=$ $1,2, \ldots, n$. The value $\mu_{i j}=\mu_{B}\left(x_{i}, x_{j}\right)$ can be interpreted as the certainty degree up to which $x_{i}$ is preferred to $x_{j}$, while the value $\nu_{i j}=\nu_{B}\left(x_{i}, x_{j}\right)$ represents the certainty degree up to which $x_{i}$ is non-preferred to $x_{j}$. When the following additional conditions are imposed:

- $\mu_{i i}=\nu_{i i}=0.5 \forall i$.

- $\mu_{j i}=\nu_{i j}, \nu_{j i}=\mu_{i j} \forall i, j$.

we refer to this IFPR as additive and we will denote it as IAPR. Notice that when the hesitancy degree function is the null function we have that $\mu_{i j}+\nu_{i j}=1 \quad \forall i, j$, and therefore the IAPR $B=\left(b_{i j}\right)$ is mathematically equivalent to the APR $\left(\mu_{i j}\right)$, i.e. $B=\left(\mu_{i j}\right)$. Given an IAPR, it is always possible to derive an APR via the application of a score function $[71,72,87]$.

\subsection{Linguistic Preferences}

Subjectivity, imprecision and vagueness in the articulation of opinions pervade real world decision applications, and individuals usually find it difficult to evaluate their preferences using exact numbers [100]. Individuals might feel more comfortable using words by means of linguistic labels or terms to articulate their preferences [101].

In a linguistic context, experts' preferences are usually represented using an ordered set of linguistic terms, $\mathcal{L}=\left\{l_{0}, \ldots, l_{s} \mid s \geq 2 \wedge i<j: l_{i}<l_{j}\right\}$. Table 1 provides an example with 
seven linguistic labels and their corresponding semantic meanings for the comparison of the ordered pair of alternatives $\left(x_{i}, x_{j}\right)$.

\begin{tabular}{|c|l|}
\hline Linguistic label & Semantic meaning \\
\hline$l_{0}$ & $x_{j}$ is absolutely preferred to $x_{i}$ \\
$l_{1}$ & $x_{j}$ is highly preferred to $x_{i}$ \\
$l_{2}$ & $x_{j}$ is slightly preferred to $x_{i}$ \\
$l_{3}$ & $x_{i}$ and $x_{j}$ are equally preferred \\
$l_{4}$ & $x_{i}$ is slightly preferred to $x_{j}$ \\
$l_{5}$ & $x_{i}$ is highly preferred to $x_{j}$ \\
$l_{6}$ & $x_{i}$ is absolutely preferred to $x_{j}$ \\
\hline
\end{tabular}

Table 1: Seven linguistic labels and their semantic meanings

An odd number of labels is also assumed, with the central label $l_{s / 2}$ standing for the indifference state when comparing two alternatives, and the remaining labels being usually located symmetrically around that central assessment to guarantees that a kind of reciprocity property holds as in the case of numerical preferences previously discussed.

Definition 8 (Linguistic Preference Relation (LPR)). A LPR P on a finite set of alternatives $X$ is characterised by a linguistic membership function $\mu_{P}: X \times X \longrightarrow \mathcal{L}, \mu_{P}\left(x_{i}, x_{j}\right)=$ $p_{i j} \in \mathcal{L}$.

The main two methodologies to manage LPRs in decision making are [36]: (i) the cardinal representation model based on the use of fuzzy sets and their associated membership functions, which are mathematically processed using Zadeh's extension principle [100]; and (ii) the ordinal representation model based on the ordered structure defined on the labels [97].

\subsubsection{LPR based on cardinal representation}

Convex normal fuzzy subsets of the real line, also known as fuzzy numbers, are commonly used to represent linguistic terms. By doing this, each linguistic assessment is represented using a fuzzy number that is characterised by a membership function, with base variable the unit interval $[0,1]$, describing its semantic meaning. The membership function maps each value in $[0,1]$ to a degree of performance which represents its compatibility with the linguistic assessment $[75,101]$.

\subsection{2. $L P R$ based on ordinal representation}

In an ordinal linguistic approach the semantics of the linguistic labels is established by assuming that in the set of linguistic terms $\mathcal{L}$ the labels are uniformly and symmetrically distributed around that central assessment $l_{s / 2}$, i.e., assuming the same discrimination levels on both sides of $l_{s / 2}$ and by considering that both terms $l_{i}$ and $l_{s-i}$ are equally informative. 
Linguistic symbolic computational models have been defined to manage the ordinal linguistic information in the decision making problems [36]. The symbolic models work with the ordinal scales of the set of linguistic terms to combine linguistic information. There exit four different linguistic symbolic computational models based on ordinal scales:

1. Linguistic symbolic computational model based on max-min operators [97], which is based on the application of the following three operators to combine information expressed as linguistic labels in the ordered linguistic set $\mathcal{L}$ :

- $\operatorname{Max}\left(l_{i}, l_{j}\right)=l_{i}$ if $l_{i}>l_{j}$.

- $\operatorname{Min}\left(l_{i}, l_{j}\right)=l_{i}$ if $l_{i}<l_{i}$.

- $N e g\left(l_{i}\right)=l_{s-i}$.

2. Linguistic symbolic model based on convex combination [37]. This model aggregates the linguistic information using a convex combination of linguistic labels acting directly over their associated indexes $\mathcal{L}$ in a recursive way. Since the result of this aggregation is not necessary integer it is also necessary to introduce an approximation function to obtain a final label in $\mathcal{L}$.

3. Linguistic symbolic model based on virtual linguistic term set [79], which extends the original discrete term set $\mathcal{L}$ into a continuous term set $\hat{\mathcal{L}}=\left\{l_{\alpha} \mid \alpha \in[-s, s]\right\}$ with the following operations :

$$
\begin{gathered}
l_{\alpha} \oplus l_{\beta}=l_{\max \{-s, \min \{\alpha+\beta, s\}\}} \\
\lambda l_{\alpha}=l_{\lambda \alpha}, \text { where } \lambda \in[0,1]
\end{gathered}
$$

This model also requires a translation function to express the results of the operations in the original terms set

4. Linguistic symbolic model based on the 2-tuple linguistic representation [38], which was introduced to avoid the loss of information that appears when the mentioned translation function in the linguistic symbolic model based on convex combination is applied. This model is built on the following linguistic 2-tuple representation definition:

Definition 9. Let $\mathcal{L}$ be a linguistic term set and $\beta \in[0, s]$ a value supporting the result of a symbolic aggregation operation, then the 2-tuple that expresses the equivalent information 
to $\beta$ is obtained with the following function:

$$
\begin{array}{ll}
\Delta: & {[0, s] \longrightarrow S \times[-0.5,0.5)} \\
& \Delta(\beta)=\left(l_{i}, \alpha\right) \\
& i=\operatorname{round}(\beta) \\
& \alpha=\beta-i
\end{array}
$$

where "round" is the usual rounding operation, $l_{i}$ has the closest index label to " $\beta$ " and " $\alpha$ " is the value of the symbolic translation.

In [58], the representation of linguistic preferences using the cardinal approach based on the use of fuzzy sets, and the ordinal approach based on the use of the 2-tuples were proved to be mathematically isomorphic when fuzzy numbers are ranked using their respective centroids.

\subsection{Consistency of Preferences}

There are three fundamental and hierarchical levels of rationality assumptions when dealing with preference relations [19]:

- The first level of rationality requires indifference between any alternative $x_{i}$ and itself.

- The second one requires that if an expert prefers $x_{i}$ to $x_{j}$, that expert should not simultaneously prefer $x_{j}$ to $x_{i}$. This asymmetry condition is viewed as an "obvious" condition/criterion of consistency for preferences [26]. This rationality condition is modelled by the property of reciprocity in the pairwise comparison between any two alternatives, which is seen by Saaty as basic in making paired comparisons [61].

- Finally, the third one is associated with the transitivity in the pairwise comparison among any three alternatives. That is, if $x_{i}$ is preferred to $x_{j}\left(x_{i} \succ x_{j}\right)$ and this one to $x_{k}$ $\left(x_{j} \succ x_{k}\right)$ then alternative $x_{i}$ should be preferred to $x_{k}\left(x_{i} \succ x_{k}\right)$, which is normally referred to as weak stochastic transitivity [49].

A preference relation verifying the third level of rationality is usually called a consistent preference relation and any property that guarantees the transitivity of the preferences is called a consistency property [19]. The lack of consistency in decision making can lead to inconsistent conclusions; that is why it is important, in fact crucial, to study conditions under which consistency is satisfied [61]. 
In the case of MPRs, Saaty means by consistency what he calls cardinal transitivity in the strength of preferences, which is a stronger condition than the traditional requirement of the transitivity of preferences [61]:

Definition 10 (Consistent MPR). A MPR A $=\left(a_{i j}\right)$ is consistent if and only if

$$
a_{i j} \cdot a_{j k}=a_{i k} \forall i, j, k=1, \ldots, n .
$$

Inconsistency for Saaty is a violation of proportionality which may not entail violation of transitivity [61]. Furthermore, consistency implies reciprocity, and therefore, they are both compatible.

For APRs, there exist many properties or conditions that have been suggested as rational conditions to be verified by a consistent relation, among which we can cite [19, 41]: triangle condition, weak transitivity, max-min transitivity, max-max transitivity, restricted max-min transitivity, restricted max-max transitivity, additive transitivity, and multiplicative transitivity. Among these, the most widely used in the context of incomplete information are the following two [19]:

Definition 11 (Additive consistency of APR [65]). An APR $P=\left(p_{i j}\right)$ on a finite set of alternatives $X$, it is additive consistent if and only if

$$
\left(p_{i j}-0.5\right)+\left(p_{j k}-0.5\right)=p_{i k}-0.5 \quad \forall i, j, k=1,2, \cdots, n
$$

Although equivalent to Saaty's consistency property for MPRs [41], additive transitivity is in conflict with the $[0,1]$ scale used for providing the preference values and therefore, it is not the most appropriate property to model consistency of reciprocal PRs.

Definition 12 (Multiplicative consistency of APR [65]). An APR $P=\left(p_{i j}\right)$ on a finite set of alternatives $X$ is multiplicative consistent if and only if

$$
p_{i j} \cdot p_{j k} \cdot p_{k i}=p_{i k} \cdot p_{k j} \cdot p_{j i} \quad \forall i, k, j \in\{1,2, \ldots n\}
$$

Multiplicative consistency property was proposed by Tanino for $p_{i j}>0 \quad \forall i, j$ and under reciprocity it is the restriction to the region $[0,1] \times[0,1] \backslash\{(0,1),(1,0)\}$ of the Cross Ratio uninorm [19]:

$$
U(x, y)= \begin{cases}0, & (x, y) \in\{(0,1),(1,0)\} \\ \frac{x y}{x y+(1-x)(1-y)}, & \text { otherwise }\end{cases}
$$


Definition 13 (Additive Consistent IVAPR $[3,69])$. An IVAPR $\widetilde{P}=\left(\widetilde{p}_{i j}\right)=\left(\left[p_{i j}^{-}, p_{i j}^{+}\right]\right)$, is additive consistent if and only if

$$
\forall i, j, k:\left\{\begin{array}{l}
p_{i k}^{-}=p_{i j}^{-}+p_{j k}^{-}-0.5 \\
p_{i k}^{+}=p_{i j}^{+}+p_{j k}^{+}-0.5
\end{array}\right.
$$

A formal approach to modelling the multiplicative consistency property of IVAPR and IAPR, however, can be found in [74].

Definition 14 (Multiplicative Consistent IVAPR [74]). An IVAPR $\widetilde{P}=\left(\widetilde{p}_{i j}\right)=\left(\left[p_{i j}^{-}, p_{i j}^{+}\right]\right)$, is multiplicative consistent if and only if

$$
\forall i, j, k:\left\{\begin{array}{l}
p_{i j}^{-} \cdot p_{j k}^{-} \cdot p_{k i}^{-}=p_{i k}^{-} \cdot p_{k j}^{-} \cdot p_{j i}^{-} \\
p_{i j}^{+} \cdot p_{j k}^{+} \cdot p_{k i}^{+}=p_{i k}^{+} \cdot p_{k j}^{+} \cdot p_{j i}^{+}
\end{array}\right.
$$

Because the IAPR $R=\left(r_{i j}\right)=\left(\left\langle\mu_{i j}, \nu_{i j}\right\rangle\right)$ is isomorphic to the IVAPR $B=\left(b_{i j}\right)=\left(\left[\mu_{i j}, 1-\right.\right.$ $\left.\left.\nu_{i j}\right]\right)$, a multiplicative consistent IAPR can be defined as follows:

Definition 15 (Multiplicative Consistent IAPR [74]). An IAPR $R=\left(r_{i j}\right)=\left(\left\langle\mu_{i j}, \nu_{i j}\right\rangle\right)$ is consistent if and only if

$$
\forall i, j, k:\left\{\begin{array}{l}
\mu_{i j} \mu_{j k} \mu_{k i}=\mu_{i k} \mu_{k j} \mu_{j i} \\
\left(1-\nu_{i j}\right)\left(1-\nu_{j k}\right)\left(1-\nu_{k i}\right)=\left(1-\nu_{i k}\right)\left(1-\nu_{k j}\right)\left(1-\nu_{j i}\right)
\end{array}\right.
$$

$\mathrm{Xu}$ et al. in [96] investigate the consistency of intuitionistic preference relations in GDM concluding that if all individual intuitionistic preference relations are consistent, then the collective intuitionistic preference relation is consistent as well. Moreover they propose an iterative approach to improve the cosistency of this type of preference relations.

In the case of LPRs, the consistency property has been defined with different expressions depending on the linguistic approach used:

Definition 16 (Cardinal Additive Consistency of LPRs [68]). Given a $L P R, \tilde{P}=\tilde{p_{i j}}$ in which each linguistic preference degree has associated a triangular fuzzy membership function, 
i.e., $\tilde{p_{i j}}=\left(p_{i j}^{L}, p_{i j}^{M}, p_{i j}^{R}\right)$, then $\tilde{P}$ is additive consistent if and only if

$$
\forall i, j, k:\left\{\begin{array}{l}
p_{i j}^{L}+p_{j k}^{L}+p_{k i}^{R}=\frac{3}{2} \\
p_{i j}^{M}+p_{j k}^{M}+p_{k i}^{M}=\frac{3}{2} \\
p_{i j}^{R}+p_{j k}^{R}+p_{k i}^{L}=\frac{3}{2}
\end{array}\right.
$$

Definition 17 (Ordinal Additive Consistency of LPRs [1]). Given a 2-tuple LPR P= $\left(p_{i j}\right)$ on a set of alternatives $X$, such that

$$
p_{i j}: X \times X \longrightarrow \mathcal{L} \times[-0.5,0.5)
$$

then $P$ will be considered consistent if for every three alternatives $x_{i}, x_{j}$ and $x_{k}$, the following condition holds

$$
p_{i k}=\Delta\left(\Delta^{-1}\left(p_{i j}\right)+\Delta^{-1}\left(p_{j k}\right)-\frac{s}{2}\right) \quad \forall i, j, k \in\{1, \ldots, n\}
$$

\subsection{Advantages and drawbacks of preference relations}

In this subsection we remark some advantages and drawbacks on the use of preference relations in decision making problems.

Millet [52] conducted a comparison study between different alternative preference elicitation methods and pairwise comparison methods were concluded to be more accurate than nonpairwise methods (utilities, orderings, ...) [27]. This is specially the case of decision making problems involving a large number of alternatives to choose from and/or conflicting and dynamic sources of information $[56,57]$. The main advantage of preference relations, which are built by pairwise comparisons, is that of focusing exclusively on two options at a time, which facilitates experts when expressing their preferences. However, the drawback is that some experts might not been able to discriminate the degree up to which some of the options are better than others, and as a consequence incomplete preferences are provided [22].

The use of different types of measurement scales to provide assessments on the alternatives lead to different preference relations: numeric or linguistic. The advantage of numeric preference relations is that of providing the preferences in a more precise way, although an associated drawback is that experts are force to assess their preferences by means of numeric assessments, obviating that some of them might feel more comfortable using words (linguistic labels) to articulate their preferences. On the other hand, linguistic preference relations are a more userfriendly representation format to express the preferences in decision making problems when 
experts' participation is necessary, and thus they are not recommended in decision making problems that not require of user-systems interaction such as automatic classification problems $[28]$.

Regarding numeric preference relations, as it was shown previously, we also have different possibilities. Crisp preference relations are the simplest and easiest to use because they are valued in the simple numerical scale $D=\left\{0, \frac{1}{2}, 1\right\}$ whose interpretation is easy to understand. However, the drawback is that of lacking flexibility to express preferences and manage uncertainty in decision making problems. To overcome this problem, APR [8] and MPR [61] were introduced, which use richer numerical scales, i.e. $D=[0,1]$ and $D=[1 / 9,9]$, respectively. Although the interpretation of intensities of preferences are different in these last two types of relation (additive interpretation vs ratio interpretation), it has been proved that are isomorphic [16], and therefore both are admissible to be used in the same problems because concepts that have been defined for APRs can be easily transpose to MPRs, and vice-versa. IVPRs [45] and IFPRs [64] were introduced to express preferences with a greater level of uncertainty in decision making problems, and it is well known that both are mathematically isomorphic. However, their drawback is twofold: experts have more difficulties in providing their preferences with such representations because more numerical parameters are to be provided, and the computation complexity of the decision making processes is higher in comparison to using APRs or MPRs.

As aforementioned, although linguistic preference relations are user-friendly and the provision of preferences by users is mitigated, they suffer the drawback of fixing the adequate linguistic scale to express preferences. Usually, we find that different experts present different conceptions to model the linguistic information and they might choose important parameters to define a linguistic modelling, such as the cardinality of linguistic term sets and the meaning associated with each label $[36,50,53]$, differently. In the case of LPRs based on cardinal representation the additional drawbacks that we find are twofold: that of defining the membership functions associated with each label and the known problem of linguistic approximation that sometimes entails loss of information [36]. Decision making approaches that use LPRs based on ordinal representation are easier to define, overcome the problem of linguistic approximation by means of the definition of symbolic computational models [37], and the problem of the loss of information by means of 2-tuple linguistic representation models can be avoided [38, 99].

\section{Decision making approaches with incomplete preferences}

It is often assumed in GDM that all the experts are able to provide preference degrees between any pair of possible alternatives, which means that complete PRs are assumed. However 
this is not always possible because of time pressure, lack of knowledge, decision maker's limited expertise on the field dealt with, or incapacity to quantify the degree of preference of one alternative over another. Thus, an expert might decide not to guess the preference values in doubt to maintain the consistency of the values already provided. To model these situations the concept of incomplete PR was introduced in [40].

Definition 18. A function $f: X \longrightarrow Y$ is partial when not every element in the set $X$ necessarily maps to an element in the set $Y$. When every element from the set $X$ maps to one element of the set $Y$ then we have a total function.

Definition 19. A preference relation $P$ on a set of alternatives $X$ with a partial membership function is an incomplete preference relation.

The concept of incomplete preference relations has attracted the attention of researchers in the past 20 years and therefore specific settings for different types of PRs have been introduced and analyse in the literature [79, 86, 93].

In this section we analyse the main techniques developed in the literature to deal with incomplete information in decision making for the different types of preference relations reviewed in Section 2. These techniques use consistency properties to estimate the missing preferences and can be divided in two different approaches:

1. Iterative approaches to estimate the missing preference values and complete the preference relations $[3,10,11,13,18,46,82]$. Some approaches also present interactive procedures to increase the consensus degree among the experts [39, 44, 82, 88, 89]

2. Optimisation approaches to estimate the missing preference values or to directly rank the alternatives without previously completing the preference relations. Therefore there are two types of these approaches:

2.1 Methods that estimate the missing preferences [25, 102], and

2.2 Methods that estimate the weighting vector $[23,30,35,48,78,81,83,88,88,94]$.

Notice that because both the iterative and the optimisation based approaches use consistency criteria, in many cases the corresponding outputs are similar, as it is proved by Chiclana et al. [17] for the case of using additive consistency property and APRs.

Figure 2 depicts a schema of the different approaches existing in the literature to deal with incomplete information in decision-making, which will be analysed in the following subsections for the case of APR and MPR, IVPR and IFPR, and LPR, respectively. 


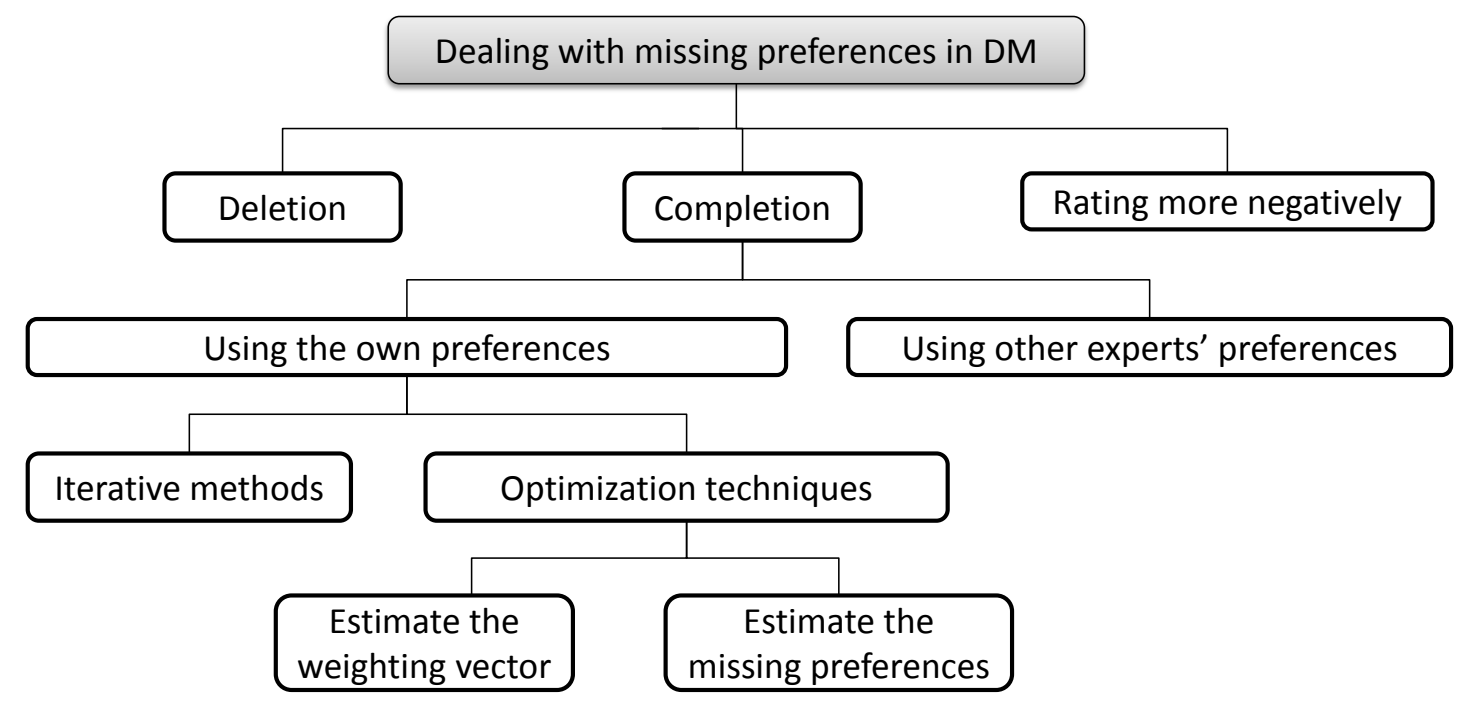

Figure 2: Different approaches to deal with missing information in DM.

\subsection{Managing missing preference values in APRs and MPRs}

Notice that the majority of the techniques developed to deal with uncertainty and missing information in GDM are for APRs and MPRs. Recall that in [16] both types of PRs were proved to be isomorphic.

\subsubsection{Iterative approaches}

Three main iterative approaches to estimate incomplete APRs and MPRs can be found: additive consistency based approaches $[3,10,11,40,46]$, multiplicative consistency based approaches [82], and its generalisation approach based on the use of uninorm operators [18].

1. Additive consistency based approaches: The main additive consistency based method is due to Herrera-Viedma et al. [40], which consists of an iterative procedure to estimate missing preference values followed by a choice process of the solution alternative. The iterative method to estimate missing preference values is summarised below:

Given an unknown preference value $p_{i j}(i \neq j)$ the iterative procedure starts by using intermediate alternatives, $x_{k}$, to create indirect chains of known preference values, $\left(p_{i k}, p_{k j}\right)$, that will be used to derive, using the additive consistency property, the local consistency based estimated values:

$$
e p_{i j}^{k}=p_{i k}+p_{k j}-0.5
$$

By averaging all the local consistency based estimated values, the overall consistency based 
estimated value is obtained:

$$
e p_{i j}=\sum_{k=1, k \neq i, j}^{n} \frac{e p_{i j}^{k}}{n-2}
$$

In each iteration, the algorithm checks the set of pairs of alternatives for which preference values are unknown and can be estimated using known ones. The algorithm stops when this set is empty. Notice that the cases when an incomplete APR cannot be successfully completed are reduced to those cases when no preference values involving a particular alternative are known, which means that a whole row or column of the APR is completely missing.

Because of the conflict between the additive consistency property and the unit scale used to measure preference values [19], the overall consistency based estimated preferences might be greater than 1 or lower than 0 , and therefore a normalisation process using the median operator is necessary [20].

In [3], an extension to deal with MPR, IVPR, and LPR is presented. The original approach by Herrera-Viedma et al. has been taken forward by many authors to tackle different research problems with incomplete APRs. Notable examples can be found in [10, 11, 39, $46]$.

2. Multiplicative consistency based approaches: The most relevant method developed using the multiplicative consistency property are presented in [82] and [90]. In [82] each individual incomplete APR is completed using the multiplicative consistency property, followed by their aggregation into a collective preference relation. Based on the deviations between the collective and individuals APRs, the decision makers interact to increase the level of consensus. In [90] it is presented a completion method for MPR based on the multiplicative transitivity. This method estimates the unknown preferences using several pairs of adjoining known elements. To compute the final value it calculates the geometrical mean of all the possible ones.

3. Uninorms based approaches: As it has been mentioned before, additive consistency property does not generalise the concept of transitivity of crisp preferences. In [19] it is shown that, under a set of conditions, consistency of APR can be characterised by representable uninorms. In [18], Herrera-Viedma et al's iterative method is adapted to implement the modelling of consistency of preferences using a self-dual almost continuous uninorm operator. Since Tanino's multiplicative transitivity property is an example of such type of uninorms $[18,19]$, this approach to deal with incomplete information in APRs is more general than the above one. 


\subsubsection{Optimisation and linear programming based methods}

The two optimisation approaches to deal with incomplete PRs are analysed next:

1. Optimisation methods to estimate missing preference values. The most relevant of these methods are due to Fedrizzi and Giove [25] and Zhang et al. [102], and they aim to estimate the missing reference values by maximizing the consistency and/or the consensus of the experts' preferences.

(a) Fedrizzi and Giove [25] propose a model that minimises the global additive inconsistency of the incomplete APR

$$
\rho=6 \cdot \sum_{i<k<j} L_{i j k}
$$

where

$$
L_{i j k}=\left(p_{i k}+p_{k j}-p_{i j}-0.5\right)^{2}
$$

The missing preference values are the variables in the global inconsistency index. A comparison between this method and Herrera-Viedma et al. [40] is reported in [17]. This study proves that both methods, driven by the additive consistency property, provide the same set of solutions for independent sets of missing comparisons but not for dependent missing comparisons. Fedrizzi and Giove's method performs worse than Herrera-Viedma et al.'s method for a large number of alternatives, and both methods fails to complete an incomplete APR when no preference values are known for at least one of the alternatives. Finally the authors conclude that both methods are complementary and therefore they introduce a new methodology for reconstructing incomplete APRs that encompasses both approaches.

(b) Zhang et al. [102] propose a model for incomplete APR $F=\left(f_{i j}\right)_{n \times n}$ that aims to calculate a complete fuzzy preference relation $F^{\prime}=\left(f_{i j}^{\prime}\right)_{n \times n}$ with $f_{i j}^{\prime}=f_{i j}$ for nonnull entries of $F$ maximising the consistency level proposed by Herrera-Viedma et al. [40]. To increase the individual consistency the following linear optimisation method that minimises the Manhattan distance between the provided preference relation and 
the completed consistent based one is proposed:

$$
\begin{aligned}
& \max C L\left(F^{\prime}\right)=1-\frac{2}{3 n(n-1)(n-2)} \sum_{i, k=1 ; j \neq k}^{n} \sum_{j=1 ; j \neq i k}^{n}\left|f_{i j}^{\prime}+f_{j k}^{\prime}-f_{i k}^{\prime}-0.5\right| \\
& \text { s.t. } f_{i j}^{\prime} \geq 0 i, j=1,2, \cdots, n \\
& \text { s.t. } f_{i j}^{\prime}+f_{j i}^{\prime}=1 i, j=1,2, \cdots, n \\
& \text { s.t. } f_{i j}^{\prime}+f_{j i}^{\prime}=1 i, j=1,2, \cdots, n \\
& \text { s.t. } f_{i j}^{\prime}=f_{i j} \text { for } f_{i j} \neq \text { null }
\end{aligned}
$$

2. Optimisation methods to directly compute the priority weights. These methods aim to rank the alternatives using directly the incomplete APR, and therefore no completion process is needed. They are based on Saaty's assumption for MPR regarding the exact functional relation between the preference values and the priority vector. Two main approaches are used to develop indirect completion models based on the computation of the priority vector: linear based methods where the unknown variables are the elements of the weighting vector $[23,35,81,83,88,94]$, and least square error minimization approaches [30, 48, 78, 88].

(a) Harker [35] extends the eigenvector approach proposed by Saaty [61] for non-negative quasi reciprocal matrices in order to apply it to the case of incomplete APRs.

(b) $\mathrm{Xu}[83]$ presents a method based on a system of equations to determine the priority vector of an incomplete APR, by replacing a missing preference value $p_{i j}$ using the following priority weighting vector based value: $\frac{w_{i}}{w_{i}+w_{j}}$. With this procedure if there exists a unique solution to this system of equations, then the obtained solution is used to rank the alternatives and to select the most desirable one; otherwise, it requires the experts to provide more evaluation information until the unique priority vector can be obtained.

(c) $\mathrm{Xu}$ and Chen [94] propose a completion method based on the additive transitivity property that requires solving a linear system of equations to rank the alternatives. Shen et al. [62] and Xu [88] subsequently proved that the relation between the original $\mathrm{PR}$ and the elements of the priority weight vector postulated by $\mathrm{Xu}$ and Chen [94], $r_{i j}=0.5\left(w_{i}-w_{j}+1\right)$, does not always hold and can lead to ambiguous priority vectors. To overcome this drawback, $\mathrm{Xu}[88]$ proposed to use the following auxiliary additive 
transitivity based APR to estimate the missing preferences values, $R^{\prime}=\left(r_{i j}^{\prime}\right)_{n \times n}$ :

$$
\begin{aligned}
r_{i j}^{\prime} & =r_{i j}, \text { if } r_{i j} \text { is known; } \\
r_{i j}^{\prime} & =\frac{n-1}{2}\left(w_{i}-w_{j}\right)+\frac{1}{2}, \text { otherwise. }
\end{aligned}
$$

(d) $\mathrm{Xu}$ [81] proposes two goal programming models for obtaining the priority vector of an incomplete APR, and their extension to obtain the collective priority vector.

(e) Dopazo and Ruiz-Tagle [23] propose a parametric goal programming model based on the consistency property of MPR to obtain the weighted priority vector. This model makes use of a dissimilarity function between the ideal case, when the preferences are consistent and there is unanimous consensus among experts, $I^{k}=\left(\frac{w_{i}}{w_{j}}\right)$, and the provided incomplete MPR, $M^{k}$. The objective function corresponds to a compromise criterion constructed as a convex combination of the two extreme criteria: to minimise the weighted sum of expert deviations and to minimise the largest weighted deviation. In this model, the relative residual aggregation is modelled by a parameter $\alpha$ used to control the importance given to the most discrepant expert.

(f) Gong [30] presented a multiplicative consistency based least-square model for APRs aiming at maximising the consensus among the experts by minimising the following error function:

$$
\begin{gathered}
\min g(w)=\sum_{i=1}^{n} \sum_{j=1}^{n} \sum_{l=1}^{d_{i j}}\left(r_{i j l} w_{j}-r_{j i l} w_{i}\right)^{2} \\
\text { s.t. } \sum_{i=1}^{n} w_{i}=1,, w_{i}>0, \quad i \in n
\end{gathered}
$$

where $d_{i j}$ stands for the number of experts who have provided a preference between the alternatives $x_{i}$ and $x_{j}$. Xu et al. [77] proposed a similar approach that accepts the following three types of incomplete PR: APR, MPR and LPRs. Similar models have been proposed based on the use of logarithmic least squares by $\mathrm{Xu}$ et al. [78] and on the additive consistency property by Liu et al. [48], respectively.

\subsection{Managing missing preference values in IVPRs an IFPRs}

In this subsection we analyse the methods proposed in the literature to deal with incomplete information when the experts' preferences are expressed by means of IVPR and IFPRs. For the case of IVPRs two main approaches are analysed: The first one uses consistency properties to estimate the missing PRs [3, 29] whereas the second one [98] is based on the rough set theory [55]. For the case of IFPR three iterative approaches have been considered [74, 87, 93]. Finally 
an approach presented by $\mathrm{Xu}$ et al. in [92] to deal with missing interval value intuitionistic additive and multiplicative preference relations (IVIFPR) is also analysed.

- Genc et al. [29] extended the optimisation method proposed by $\mathrm{Xu}$ and Chen $\mathrm{Xu}$ and Chen [95] for deriving the priority weighting vector to the case of incomplete IVPRs. To that aim they also propose the so-called interval multiplicative transitivity property.

- Alonso et al. [3] extend the iterative procedure proposed by Herrera-Viedma et al. [40] to the case of IVPR relations.

- Yang et al. [98] propose a dominance-based rough set approach to estimate missing values in incomplete interval-valued information systems. This approach considers three types of unknown values: (i) IVPR with unknown upper limit and known lower limit, (ii) IVPR with unknown lower limit and known upper limit, and (iii) IVPR data with both unknown lower and upper limits.

- Xu [87] firstly defines the concept of IFPR and introduces an iterative completion method based on the multiplicative consistency.

- Xu et al. [93] presents a completion method based on the multiplicative consistency property for IFPR. This method can be summarised as follows:

Given an incomplete IFPR $R=\left(r_{i j}\right)_{n \times n}$ each missing preference value $r_{i j}(i=1,2, \cdots, n-$ $1, j=i+1)$ is estimated by $\dot{r}_{i j}=\left(\dot{\mu}_{i j}, \dot{v}_{i j}, \dot{\pi}_{i j}\right)$ where

$$
\begin{aligned}
& \dot{\mu}_{i j}=\frac{1}{m_{i j}} \sum_{k \in M_{i j}} \frac{\mu_{i k} \mu_{k j}}{\mu_{i k} \mu_{k j}+\left(1-\mu_{i k}\right)\left(1-\mu_{k j}\right)} \\
& \dot{v}_{i j}=\frac{1}{m_{i j}} \sum_{k \in M_{i j}} \frac{v_{i k} v_{k j}}{v_{i k} v_{k j}+\left(1-v_{i k}\right)\left(1-v_{k j}\right)} \\
& \text { for all } r_{i k}, r_{k j} \in \Omega, \text { and } i \leq k \leq j
\end{aligned}
$$

and $\pi_{i j}=1-\dot{\mu_{i j}}-\dot{v_{i j}}$, where $r_{i k}=\left(\mu_{i j}, v_{i j}, \pi_{i j}\right)$, and $\Omega$ is the set of all the known elements in $\mathrm{R}, M_{i j}=\left\{k \mid r_{i k}, r_{k j} \in \Omega\right\}$ and $m_{i j}$ is the number of elements in $M_{i j}$. If there exists $k_{0}$ such that $\left(\mu_{i k_{0}}, \mu_{i k_{0}}\right) \in\{(0,1),(1,0)\}$ or $\left(v_{k_{0} j}, v_{k_{0} j}\right) \in\{(0,1),(1,0)\}$, then $\frac{\mu_{i k} \mu_{k j}}{\mu_{i k} \mu_{k j}+\left(1-\mu_{i k}\right)\left(1-\mu_{k j}\right)}=0$

- Wu and Chiclana [74] propose a GDM process with consensus in which the missing values of the IFPR are estimated following an iterative procedure that is based on the one proposed by Herrera-Viedma et al. [40]. This method is based on the multiplicative 
consistency property for IFPRs, which is formally generalised from APR to IFPR by applying Zadeh's Extension Principle [101] and Representation Theorem [100] .

- Xu et al introduce in [92] the additive and the multiplicative consistent incomplete intervalvalued intuitionistic fuzzy preference relations and define the concept of acceptable incomplete interval-valued intuitionistic fuzzy preference relation. In this contribution they also propose two procedures for completing the acceptable incomplete interval-valued intuitionistic based on the arithmetic average and the geometric mean, respectively.

- Wang et al. [70] propose an approach to multiattribute decision making with incomplete attribute weight information where individual assessments are provided as IVIFPRs. By employing a series of optimization models, the proposed approach derives a linear program for determining attribute weights

\subsection{Managing missing preference values in LPRs}

There are three different methodologies to deal with incomplete LPRs, which are defined according to the three different linguistic decision frameworks: (i) 2-tuple LPRs [3, 12, 59]; (ii) LPRs based on virtual linguistic term sets $[42,85]$; and (iii) LPRs based on a cardinal approach $[47,68]$.

(i) 2-tuple LPRs.

(a) Alonso et al. [3] propose a method which converts the 2- tuple LPR into an APR and estimates the missing values using the additive transitivity property. Once the APR is completed it is transformed back to the corresponding 2-tuple LPR.

(b) Alonso et al. [1] apply the linguistic additive consistency property to estimate the missing 2-tuple linguistic values and design an iterative procedure similar to the one proposed by Herrera-Viedma et al. [40]. That was later used by Cabrerizo et al. [12] to define an additive consistency measure of the information provided by each expert to assign importance degrees to experts in the aggregation process. Porcel and Herrera-Viedma [59] present an application in the context of fuzzy linguistic recommender systems that allows incomplete linguistic information.

(ii) LPRs based on Virtual linguistic term sets.

(a) $\mathrm{Xu}[85]$ proposes an additive transitivity property based method to estimate missing LPRs assessed on virtual linguistic term sets. This author also propose in [84] and in [76] completion methods based on the multiplicative transitivity. 
(b) Hsu and Wang [42] present an alternative additive transitivity property based estimation method of missing LPRs assessed on virtual linguistic term sets for which they propose three ways of pairwise comparisons: horizontal, vertical and oblique.

(iii) LPRs based on a cardinal approach.

(a) Li and Sun [47] propose an extension of the well known LINMAP method [63] to deal with decision making problems with fuzzy linguistic information. Each alternative is assessed on the basis of its distance to a fuzzy positive ideal solution (FPIS) which is unknown, using a new method to calculate the distance between trapezoidal fuzzy number scores. The FPIS and the weights of attributes are then estimated using a linear programming model guided by the consistency and inconsistency criteria. The distance of each alternative to the FPIS is calculated to determine the ranking order of all alternatives.

(b) Wang and Chen [68] present an approach which uses triangular membership function to model linguistic information and that is driven by the additive consistency property of the reciprocal APR.

\subsection{Summary}

Table 2 summarises, in chronological order, the main papers dealing with the different approaches to manage incomplete information reviewed and analysed in this contribution. It is fair to conclude that the management of incomplete information in DM based on PRs is currently a relevant topic in fuzzy decision making analysis, and that it has been disseminated in the most important journals on this research area including: IEEE Trans. on Systems, Man and Cybernetics-Part B; IEEE Trans. on Fuzzy Systems; Knowledge-Based Systems; Information Sciences; Information Fusion, Soft Computing and Fuzzy Sets and Systems. Evidence of this is that the scientific database Essential Science Indicators, provided by Thomson Reuter, is currently listing incomplete information as part of the following Research Front: Incomplete Fuzzy Linguistic Preference Relations; Group Consensus Algorithm Based; Unbalanced Fuzzy Linguistic Information; AHP Group Decision (accessed on 29-10-2014).

\section{Processes dealing with ignorance situations in GDM}

The procedures exposed in the previous section cannot be applied successfully when some experts do not provide any information about a particular alternative, which is known as ignorance situations. Alonso et al. [4] developed several strategies to deal with ignorance situations 


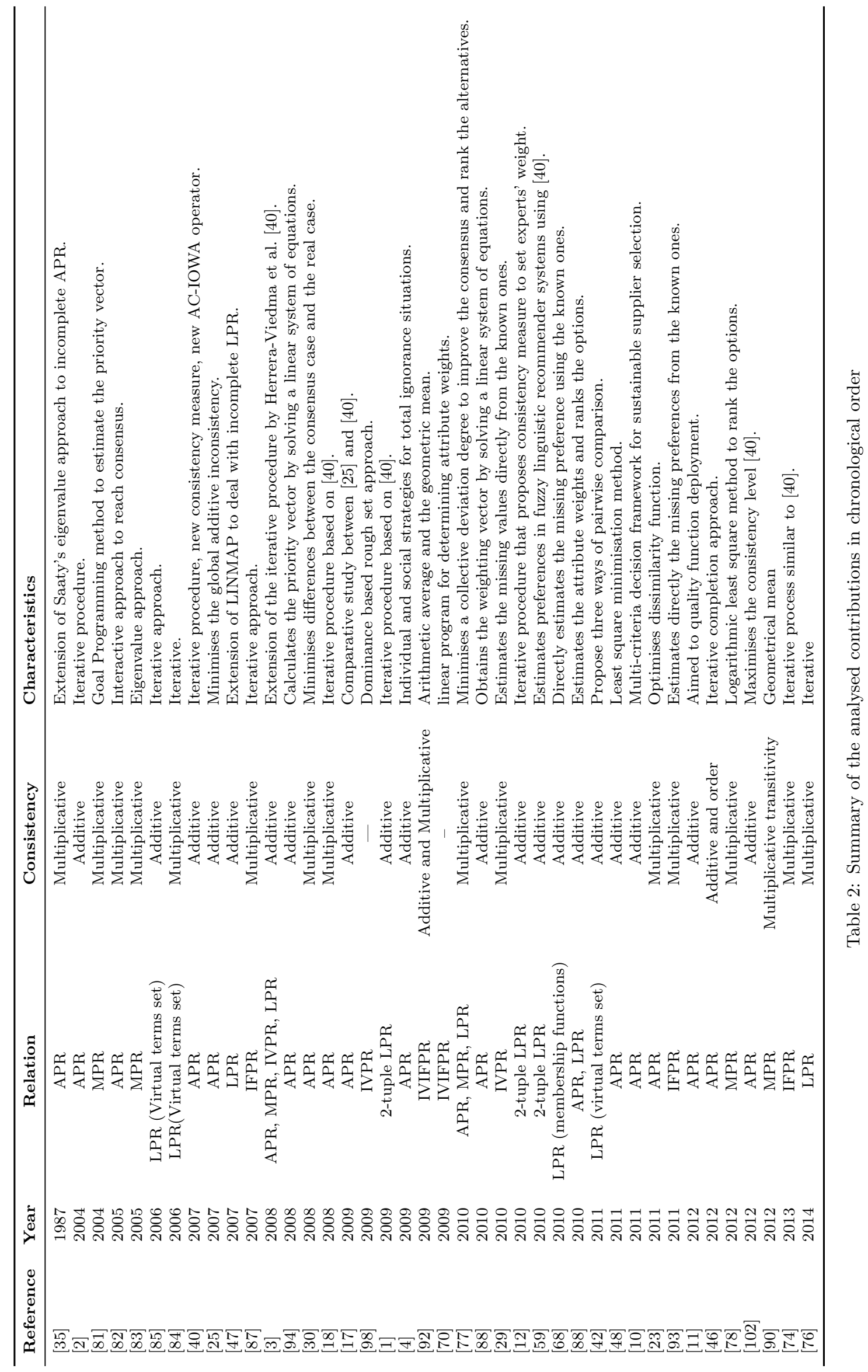


in the context of GDM with APRs. These strategies can be broadly classified in two main groups depending on whether the information provided by other experts is used to estimate the missing values, known as social strategies, otherwise named individual strategies.

\subsection{Ignorance individual strategies}

The proposed ignorance individual strategies (IIS) can be divided in two main steps:

1. Setting some particular seed values to provide some initial information to the estimation procedure to be able to compute the other missing values. The selection of the seed values can be accomplished using two different methodologies:

IIS1 Choosing indifference seed values: Let P be an incomplete APR with no preference information on alternative $x_{i}$, i.e $p_{i j}^{\prime}$ and $p_{j i}^{\prime}$ are unknown for all $j$. In this strategy, indifference seed values are assumed, i.e. $p_{i j}^{\prime}=p_{j i}^{\prime}=0.5 \forall j$. This strategy adjusts the estimated preference values to make the APR more consistent with the previously existing information. This approach is particularly useful when there are no external sources of information about the problem and when a high consistency level is required.

IIS2 Choosing proximity seed values: In this case the seed values are obtained from the preference values given to similar alternatives. This is possible if some extra information or properties about alternatives, which strongly suggest that the ignored alternative is similar to another one, are known. This strategy could be useful in some decision making problems where the alternatives to be evaluated are goods with similar characteristics (similar models).

2. Estimating the rest of the missing values using the consistency based procedure proposed in $[40]$.

\subsection{Ignorance social strategies}

Ignorance social strategies (ISS) are based on the use of the information provided by the set of experts. The authors present three main approaches in this case:

ISS1 The first social strategy uses consensus preference values of the collective PR, computed by aggregating all the experts' individual PRs. The main advantage of this approach is that it improves the consensus of the set of experts making their opinions close to each other. 
ISS2 The second strategy uses only the consensus preference values provided by those experts nearest to the expert whose $\mathrm{PR}$ is incomplete. This strategy is aimed to narrow the differences between the expert with an ignored alternative and those who have a similar opinion about the rest of alternatives.

ISS3 The third approach integrates the previous two by taking into account both information from the collective preference relation and from the nearest experts. This strategy encompasses the advantages of the previous two social strategies since the estimated information not only helps in the consensus process but also tries to keep a high consistency level in the individual experts' PR. Therefore it is considered by the authors of the proposal as the best strategy to deal with ignorance situations in GDM.

\subsection{Advantages and drawbacks of ignorance strategies}

In this section we will discuss the advantages and drawbacks of each one of the five strategies, and the situations where some of them may be more adequate to be applied than the others.

IIS1 This strategy improves the approach which considers ignorance equivalent to indifference because the initial indifference preference values associated to the unknown alternative is corrected, by means of the consistency property, when there is no indifference between some of the other alternatives. This approach is particularly useful when there are no external sources of information about the problem and when a high consistency level is required in the experts' preference relations.

IIS2 This strategy implies having some additional knowledge about the alternatives of the problem, and as such it is recommended to be use in decision problems where the alternatives to be evaluated share similar characteristics (similar models), which can be exploited to avoid ignorance situations in which an expert is not familiar with one of the alternatives, but has enough knowledge about a similar one.

ISS1 This strategy is appropriate for GDM problems because their resolution process usually requires the computation of the collective preference relation, and it could help to reach a consensus more easily because the unknown preferences are estimated from the collective ones. Additionally, the use of the estimation procedure assures that the loss of consistency will be minimized. Thus, this kind of approach could be useful in problems where a fast and converging consensus process is needed.

ISS2 This strategy also helps the consensus process to converge because an expert's unknown information is obtained from the nearest experts. However, this convergence is achieved in 
a different way with respect to the previous social strategy because the unknown information is estimated by using only the information of some of the experts. This strategy could prove useful in GDM problems in which the estimated information should be compatible with the information expressed by the expert, which is assured because it is obtained using the information of the nearest experts rather than the information from the whole group of experts.

ISS3 This strategy unifies all the advantages of the previous two social strategies. The estimated information will not only help in the consensus process to converge but also will try to maintain a high consistency level for the expert.

\section{Trends and future work}

According to the previous analysis we present some current trends on the estimation of information in GDM, along with some open questions and prospects about them. We identify three current trends:

1. Development of management procedures of incomplete preferences in the case of hesitant and type-2 PRs.

2. Development of comparison tools to evaluate and validate the different GDM approaches.

3. Managing incomplete information in Web 2.0 contexts.

5.1. Development of management procedures of incomplete preferences in the case of hesitant and type-2 PR.

Experts can perceive the provision of preferences for real decision making processes as complex because of the multiple alternatives and criteria that they need to be taken into account. Therefore it is natural that experts might present some degree of hesitancy in the expression of their preferences. To that aim the use of preference relations such us the IFPR and the IVPR are attracting the attention of many researchers in the last decade. Additionally, two very promising types of PRs are becoming recently widely used in decision making as well: (i) type-2 fuzzy PR [51] (ii) hesitant fuzzy PRs (HFPR) [66]

The concept of HFPR is captured in the following [91]:

Definition 20. Let $X=\left\{x_{1}, s_{2}, \cdots, x_{n}\right\}$ be a fixed set, a HFPR $H$ on $X$ is presented by a matrix $H=\left(h_{i j}\right)_{n x n} \subset X \times X$ where $h_{i j}=\left\{h_{i j}^{s}, s=1,2, \cdots, l_{h_{i j}}\right\}$ is a HFS indicating al 
the possible degrees to which $x_{i}$ is preferred to $x_{j}$. Moreover, $h_{i j}$ should satisfy the following conditions:

$$
h_{i j}^{\sigma(s)}+h_{j i}^{\sigma\left(l_{h_{i j}}-s\right)+1}=1, h_{i i}=\{0.5\}, l_{h_{i j}}=l_{h_{j i}}, i, j=1,2, \cdots, n
$$

The use of HFPRs in GDM have attracted the attention of many researchers in the last few years $[91,103]$. However, as far as we are aware, there is no approach in the literature able to deal with incomplete HFPRs. A possible approach in these cases would be to extend existing validated approaches for the case of incomplete APRs, IVPRs, IFPRs and LPRs using the multiplicative consistency property of HFPRs introduced in [103] and the iterative procedure developed in [19]. However, it remains to be developed a formal and theoretical sound framework to support the validity of the methodology adopted in this area, which consists of the straightforward application of existing mathematical tools and procedure developed specifically for type-1 fuzzy preferences to hesitancy preferences. Without tackling this issue in the first place it could well be that some of the approaches already proposed in this area could well been proved to be incorrect, as it has been already the case of the modelling of multiplicative consistency in the case of intuitionistic preference relations put forward in [87] that has been subsequently proved to be incorrect by the author proposing it and others in [74]. A possible avenue to investigate to tackle this issue might reside in the similarities that exist between the definitions of hesitant fuzzy set and that of type-2 fuzzy set, which can lead to considering the first one as a particular type of the second one. In any case, type-2 PRs, i.e. preference relations whose elements are type-2 fuzzy sets, have not been the object of research regarding the estimation of missing type-2 fuzzy preference values in decision making. An explanation for this might reside in the complexity of type-2 computation. In any case, a possible approach to develop in this case would necessarily involve the decomposition of each type-2 fuzzy sets in its associated set of type-1 embedded sets to which type-1 fuzzy approaches are possible to be applied in conduction to the application of Zadeh's extension principle to obtain the type-2 fuzzy set output [21, 31-34].

5.2. Development of comparison tools to evaluate and validate decision making approaches with incomplete preferences

It is clear that there are many different decision making approaches to tackle incomplete information. However, it is also evident that there is a lack of a comparison framework available to evaluate their performance and consequently to help analyse the causes that might affect such performance. This shortage of comparison tools represent an important problem in the decision making field because decision making practitioners are unable to discriminate between the accuracy and the quality of the proposals available to them in the context of incomplete 
information. Thus, it seems imperative to develop methods to evaluate and validate the different techniques proposed in the literature to estimate the missing preferences. By doing this, it could be possible to compare in a quantitative way the existing GDM methodologies and find out which ones are more suitable depending on the problem to solve and to identify their main advantages and drawbacks.

Some initial efforts in the direction pointed above have been presented in [9] and in [15]. Brunelli et al. [9] conducted a comparative study of seven different methods for reconstructing incomplete fuzzy preference relations in terms of the consistency of the resulting complete preference relation; while Chiclana et al. [15] carried out a statistical comparative study to find out the differences in group consensus that different distance measures could lead to.

However the development of methods to evaluate the quality of the different GDM approaches with incomplete preferences is still in a very early stage and therefore there are many challenges that need to be addressed:

- To create a training and test framework with examples to allow benchmark tests to compare and validate different decision making approaches.

- To find proper metrics to compare different completion approaches.

- To develop software tools to carry out the evaluation and comparison of the different GDM approaches in the literature.

\subsection{Managing incomplete information in the new Web 2.0 contexts.}

Web 2.0 is the common term for advanced internet technologies and applications including social networks, blogs, wikis, RSS, podcasting and mashups. Web 2.0 content is user generated and it is characterised for the high degree of collaboration among internet users. As a result, these technologies provide an ideal framework to collaborate, negotiate, communicate, and interact while at the same time allowing their users to take advantage of values such as democratic participation, collaboration, collective intelligence and knowledge sharing on a massive scale beyond geographical barriers. All these values are extremely useful in social decision making processes [67]. Thus, it becomes necessary to adapt and develop new and appropriate decision making approaches for these new environments. In any case, it is of special importance to be aware that web 2.0 communities have some peculiarities, among which the most relevant to the efforts in developing tailored decision making models are [6]: (i) it constitutes a large and heterogeneous user base expressing opinions and preferences; and (ii) the low and intermittent participation rate. 
Initial research proposals in this area can be found in [6, 59]. Alonso et al. [6] described a consensus approach for web 2.0 technologies, which includes a delegation feedback; while Porcel and Herrera-Viedma [59] developed a method to estimate users' preferences in fuzzy linguistic recommender system. However, due to the inherent characteristics of the Web 2.0 communities in many occasions the information about users' preferences is scarce or incomplete. These situations provide a good opportunity to implement incomplete preference management procedures in web 2.0 context: (1) to extend some of the previous incomplete approaches here reviewed; and (2) to develop new mechanisms to estimate missing information based on new information inherent to web 2.0 context such as trust degree, reputation or new techniques based on social networks analysis [73].

\section{Conclusions}

In decision making, situations where all experts are able to efficiently express their preferences over all the available options are the exception rather than the rule. Indeed, the above scenario requires from all experts to possess a precise or sufficient level of knowledge of the whole problem to tackle, including the ability to discriminate the degree up to which some options are better than others, which can obviously seen as unrealistic in many decision making situations, especially those involving a considerable large number of alternatives to choose from and/or conflicting and dynamic sources of information.

In this paper we have reviewed and analysed the state-of-the-art research efforts on group decision making from the perspective of the estimation of missing preferences using different types of preference relations. We have presented the foundations and developments in that field along with the most relevant computational models that have been applied to the decision making context: APR, MPR, IFPR, IVPR and LPR. These estimation techniques mainly use the additive or the multiplicative consistency properties to calculate the missing preferences from the known ones, as well as increasing the global consistency level and in many cases the experts' consensus. They can be widely classified in two main groups: (i) iterative procedures, and (ii) optimisation procedures. A comprehensive list of the most recent developed applications in the specialised literature has been presented. Finally, several current trends and prospects about the topic have been introduced.

\section{Acknowledgments}

This research work has been developed with the financing of the research projects TIN201017876, TIN2013-40658-P, TIC-05299, TIC-5991, and the mobility grant program awarded in 
2012 by the University of Granada's International Office.

\section{References}

[1] Alonso, S., Cabrerizo, F., Chiclana, F., Herrera, F., Herrera-Viedma, E., 2009. Group decision making with incomplete fuzzy linguistic preference relations. International Journal of Intelligent Systems 24 (2), 201-222.

[2] Alonso, S., Chiclana, F., Herrera, F., Herrera-Viedma, E., 2004. A learning procedure to estimate missing values in fuzzy preference relations based on additive consistency. Lecture Notes in Artificial Intelligence 3131, 227-238.

[3] Alonso, S., Chiclana, F., Herrera, F., Herrera-Viedma, E., Alcalá-Fdez, J., Porcel, C., 2008. A consistency-based procedure to estimate missing pairwise preference values. International Journal of Intelligent Systems 23 (2), 155-175.

[4] Alonso, S., Herrera-Viedma, E., Chiclana, F., Herrera, F., 2009. Individual and social strategies to deal with ignorance situations in multi-person decision making. International Journal of Information Technology and decision Making 8 (2), 313-333.

[5] Alonso, S., Herrera-Viedma, E., Chiclana, F., Herrera, F., 2010. A web based consensus support system for group decision making problems and incomplete preferences. Information Sciences 180 (23), 4477-4495.

[6] Alonso, S., Pérez, I., Cabrerizo, F., Herrera-Viedma, E., 2013. A linguistic consensus model for web 2.0 communities. Applied Soft Computing 13 (1), 149-157.

[7] Atanassov, K. T., 1986. Intuitionistic fuzzy sets. Fuzzy Sets and Systems 20 (1), 87-96.

[8] Bezdek, J., Spillman, B., R., R. S., 1978. A fuzzy relation space for group decision theory. Fuzzy Sets and Systems 1 (4), 255-268.

[9] Brunelli, M., Fedrizzi, M., Giove, S., 2007. Reconstruction methods for incomplete fuzzy preference relations: A numerical comparison. In: Proceedings of the 7th international workshop on Fuzzy Logic and Applications: Applications of Fuzzy Sets Theory. WILF '07. Springer-Verlag, pp. 86-93.

[10] Buyukozkan, G., Cifci, G., 2011. A novel fuzzy multi-criteria decision framework for sustainable supplier selection with incomplete information. Computers in Industry 62 (2), $164-174$. 
[11] Buyukozkan, G., Cifci, G., 2012. A new incomplete preference relations based approach to quality function deployment. Information Sciences 206, 30-41.

[12] Cabrerizo, F. J., Heradio, R., Pérez, I. J., Herrera-Viedma, E., 2010. A selection process based on additive consistency to deal with incomplete fuzzy linguistic information. Journal of Universal Computer Science 16 (1), 62-81.

[13] Cabrerizo, F. J., Pérez, I. J., Herrera-Viedma, E., 2010. Managing the consensus in group decision making in an unbalanced fuzzy linguistic context with incomplete information. Knowledge-Based Systems 23 (2), 169-181.

[14] Carmone, F. J., Kara, A., Zanakis, S. H., 1997. A monte carlo investigation of incomplete pairwise comparison matrices in AHP. European Journal of Operational Research 102 (3), $538-553$.

[15] Chiclana, F., García, J. T., del Moral, M., Herrera-Viedma, E., 2013. A Statistical comparative study of different similarity measures of consensus in group decision making. Information Sciences 221, 110-123.

[16] Chiclana, F., Herrera, F., Herrera-Viedma, E., 2001. Integrating multiplicative preference relations in a multipurpose decision-making model based on fuzzy preference relations. Fuzzy Sets and Systems 122 (2), 277-291.

[17] Chiclana, F., Herrera-Viedma, E., Alonso, S., 2001. A note on two methods for estimating missing pairwise preference values. IEEE Transactions on Systems, Man, and Cybernetics, Part B: Cybernetics 39 (6), 1628-1633.

[18] Chiclana, F., Herrera-Viedma, E., Alonso, S., Herrera, F., 2008. A note on the estimation of missing pairwise preference values: a uninorm consistency based method. International Journal of Uncertainty, Fuzziness and Knowledge-Based Systems 16 (2), 19-32.

[19] Chiclana, F., Herrera-Viedma, E., Alonso, S., Herrera, F., 2009. Cardinal consistency of reciprocal preference relations: A characterization of multiplicative transitivity. IEEE Transactions on Fuzzy Systems 17 (1), 14-23.

[20] Chiclana, F., Mata, F., Martínez, L., Alonso, S., Herrera-Viedma, E., 2008. Integration of a consistency control module within a consensus decision making model. International Journal of Uncertainty, Fuzziness and Knowledge-Based Systems 16 (1), 35-53.

[21] Chiclana, F., Zhou, S.-M., 2013. Type-reduction of general type-2 fuzzy sets: the type-1 owa approach. International Journal of Intelligent Systems 28 (5), 505-522. 
[22] Deparis, S., Mousseau, V., Ozturk, M., Pallier, C., Huron, C., 2012. When conflict induces the expression of incomplete preferences. European Journal of Operational Research $221(3), 593-602$.

[23] Dopazo, E., Ruiz-Tagle, M., 2011. A parametric gp model dealing with incomplete information for group decision-making. Applied Mathematics and Computation 218 (2), 514-519.

[24] Ebenbach, D. H., Moore, C., 2000. Incomplete information, inferences, and individual differences: The case of environmental judgments. Organizational Behavior and Human Decision Processes 81 (1), 1-27.

[25] Fedrizzi, M., Giove, S., 2007. Incomplete pairwise comparison and consistency optimization. European Journal of Operational Research 183 (1), 303-313.

[26] Fishburn, P., 1979. Utility theory for decision making. Krieger, Melbourne, FL.

[27] Fodor, J., Roubens, M., 1994. Fuzzy preference modelling and multicriteria decision support. Kluwer Academic Publishers.

[28] Galar, M., Fernandez, A., Barrenechea, E., Herrera, F., 2014. Empowering difficult classes with a Similarity-based aggregation in multi-class classification problems. Information Sciences 264, 135-157

[29] Genc, S., Boran, F. E., Akay, D., Xu, Z., 2010. Interval multiplicative transitivity for consistency, missing values and priority weights of interval fuzzy preference relations. Information Sciences 180 (24), $4877-4891$.

[30] Gong, Z.-W., 2008. Least-square method to priority of the fuzzy preference relations with incomplete information. International Journal of Approximate Reasoning 47 (2), 258-264.

[31] Greenfield, S., Chiclana, F., 2013. Accuracy and complexity evaluation of defuzzification strategies for the discretised interval type-2 fuzzy set. International Journal of Approximate Reasoning 54 (8), 1013-1033.

[32] Greenfield, S., Chiclana, F., 2013. Defuzzification of the discretised generalised type-2 fuzzy set: experimental evaluation. Information Sciences 224, 1-25.

[33] Greenfield, S., Chiclana, F., John, R., Coupland, S., 2012. The sampling method of defuzzification for type-2 fuzzy sets: experimental evaluation. Information Sciences 189, 77-92.

[34] Greenfield, S., Chiclana, F., Coupland, S., John, R., 2009. The collapsing method of defuzzification for discretised interval type-2 fuzzy sets. Information Sciences 179(13), 2055-2069. 
[35] Harker, P., 1987. Alternative modes of questioning in the analytic hierarchy process. Mathematical Modelling 9 (3-5), 353-360.

[36] Herrera, F., Alonso, S., Chiclana, F., Herrera-Viedma, E., 2009. Computing with words in decision making: foundations, trends and prospects. Fuzzy Optimization and Decision Making 8 (4), 337-364.

[37] Herrera, F., Herrera-Viedma, E., Verdegay, J. L., 1996. Direct approach processes in group decision making using linguistic owa operators. Fuzzy Sets and Systems (79), 175-190.

[38] Herrera, F., Martinez, L., 2000. A 2-tuple fuzzy linguistic representation model for computing with words. IEEE Transactions on Fuzzy Systems 8, 746-752.

[39] Herrera-Viedma, E., Alonso, S., Chiclana, F., F.Herrera, 2007. A consensus model for group decision making with incomplete fuzzy preference relations. IEEE Transactions on Fuzzy Systems 15 (5), 863-877.

[40] Herrera-Viedma, E., Chiclana, F., F.Herrera, Alonso, S., 2007. Group decision-making model with incomplete fuzzy preference relations based on additive consistency. IEEE Transactions on Systems, Man, and Cybernetics, Part B: Cybernetics 37 (1), 176-189.

[41] Herrera-Viedma, E., Herrera, F., Chiclana, F., Luque, M., 2004. Some issues on consistency of fuzzy preference relations. European Journal of Operational Research 154 (1), 98-109.

[42] Hsu, S.-C., Wang, T.-C., 2011. Solving multi-criteria decision making with incomplete linguistic preference relations. Expert Systems with Applications 38 (9), 10882-10888.

[43] Kabak, O., Ruan, D., 2011. A cumulative belief degree-based approach for missing values in nuclear safeguards evaluation. IEEE Transactions on Knowledge and Data Engineering, 23 (10), 1441-1454.

[44] Kim, J. K., Choi, S. H., Han, C. H., Kim, S. H., 1998. An interactive procedure for multiple criteria group decision making with incomplete information. Computers and Industrial Engineering 35 (1-2), 295-298.

[45] Klir, G. J., Folger, T. A., 1992. Fuzzy sets, uncertainty, and information. Prentice-Hall International.

[46] Lee, L.-W., 2012. Group decision making with incomplete fuzzy preference relations based on the additive consistency and the order consistency. Expert Systems with Applications 39 (14), 11666-11676. 
[47] Li, D.-F., Sun, T., 2007. Fuzzy linmap method for multiattribute group decision making with linguistic variables and incomplete information. International Journal of Uncertainty, Fuzziness and Knowledge-Based Systems 15 (2), 153-173.

[48] Liu, X., Pan, Y., Xu, Y., Yu, S., 2012. Least square completion and inconsistency repair methods for additively consistent fuzzy preference relations. Fuzzy Sets and Systems $198(1), 1-19$.

[49] Luce, R. D., Suppes, P., 1965. Preferences, utility and subject probability. Handbook of mathematical psychology. Vol. 3. New York.

[50] Massanet, S., Riera, J.V., Torrens, J., Herrera-Viedma, E., 2013. A new linguistic computational model based on discrete fuzzy numbers for computing with words. Information Sciences 258, 277-290.

[51] Mendel, J. M., 2001. Uncertain rule-based fuzzy logic systems: introduction and new directions. Prentice-Hall PTR.

[52] Millet, I., 1997. The effectiveness of alternative preference elicitation methods in the analytic hierarchy process. Journal of Multi-Criteria Decision Analysis 6 (1), 41-51.

[53] Morente-Molinera, J.A., Perez, I.J., Ureña, R., Herrera-Viedma, E., 2015. On multigranular fuzzy linguistic modelling in group decision making problems: a systematic review and future trends Knowledge-Based Systems 74, 49-60.

[54] Orlovsky, S., 1978. Decision-making with a fuzzy preference relation. Fuzzy Sets and Systems $1(3), 155-167$.

[55] Pawlak, Z., 1992. Rough sets: theoretical aspects of reasoning about data. Kluwer Academic Publishers.

[56] Pérez, I.J., Cabrerizo, F.J., Herrera-Viedma, E., 2010. A Mobile Decision Support System for Dynamic Group Decision Making Problems. IEEE Transactions on Systems, Man and Cybernetics - Part A: Systems and Humans 40 (6),1244-1256.

[57] Pérez, I.J., Cabrerizo, F.J., Alonso, S., Herrera-Viedma, E., 2014. A New Consensus Model for Group Decision Making Problems with Non Homogeneous Experts. IEEE Transactions on Systems, Man, and Cybernetics: Systems 44 (4), 494-498.

[58] Pérez-Asurmendi, P., Chiclana, F., 2014. Linguistic majorities with difference in support. Applied Soft Computing 18, $196-208$. 
[59] Porcel, C., Herrera-Viedma, E., 2010. Dealing with incomplete information in a fuzzy linguistic recommender system to disseminate information in university digital libraries. Knowledge-Based Systems 23, 32-39.

[60] Roubens, M., Vincke, P., 1985. Preference modeling. Springer, Berlin.

[61] Saaty, T. L., 1980. The analytic hierarchy process. McGraw-Hill.

[62] Shen, P.-D., Chyr, W.-L., Lee, H.-S., Lin, K., 2009. Correspondence between incomplete fuzzy preference relation and its priority vector. In Proceedings of the 13th International Conference KES ’09. Springer-Verlag, Berlin, Heidelberg, pp. 745-751.

[63] Srinivasan, V., Shocker, A., 1973. Linear programming techniques for multidimensional analysis of preference. Psychometrika 38, 337-342.

[64] Szmidt, E., Kacprzyk, J., 2002. Using intuitionistic fuzzy sets in group decision making. Control and Cybernetics 31 (4), 1037-1053.

[65] Tanino, T., 1984. Fuzzy preference orderings in group decision making. Fuzzy Sets and System 12, 117-131.

[66] Torra, V., 2010. Hesitant fuzzy sets. International Journal of Intelligent Systems 25, 529539.

[67] Turban, E. Liang, T.-P., Wu, S. P. J., 2011. A framework for adopting collaboration 2.0 tools for virtual group decision making. Group Decision and Negotiation 20 (2), 137-154.

[68] Wang, T.-C., Chen, Y.-H., 2010. Incomplete fuzzy linguistic preference relations under uncertain environments. Information Fusion 11 (2), 201-207.

[69] Wang, Y.-M., Yang, J.-B., Xu, D.-L., 2005. A two-stage logarithmic goal programming method for generating weights from interval comparison matrices. Fuzzy Sets and Systems $152(3), 475-498$.

[70] Wang, Z., Li, K. W., Wang, W., 2009. An approach to multiattribute decision making with interval-valued intuitionistic fuzzy assessments and incomplete weights. Information Sciences 179 (17), $3026-3040$.

[71] Wu, J., Chiclana, F., 2012. Non-dominance and attitudinal prioritisation methods for intuitionistic and interval-valued intuitionistic fuzzy preference relations. Expert Systems with Applications 39 (18), 13409-13416. 
[72] Wu, J., Chiclana, F., 2014. A risk attitudinal ranking method for interval-valued intuitionistic fuzzy numbers based on novel score and accuracy expected functions. Applied Soft Computing 22, 2014, 272-286.

[73] Wu, J., Chiclana, F., 2014. A social network analysis trust-consensus based approach to group decision-making problems with interval-valued fuzzy reciprocal preference relations. Knowledge-Based Systems 59, 2014, 97-107.

[74] Wu, J., Chiclana, F., 2014. Multiplicative consistency of intuitionistic reciprocal preference relations and its application to missing values estimation and consensus building. Knowledge-Based Systems, 71, 187-200.

[75] Wu, J., Chiclana, F., 2014. Visual information feedback mechanism and attitudinal prioritisation method for group decision making with triangular fuzzy complementary preference relations. Information Sciences 279, 716-734.

[76] Xia, M., Xu, Z., Wang, Z., 2014. Multiplicative consistency-based decision support system for incomplete linguistic preference relations. International Journal of Systems Science $45(3), 625-636$.

[77] Xu, Y., Da, Q., Wang, H., 2010. A note on group decision-making procedure based on incomplete reciprocal relations. Soft Computing 15 (7), 1289-1300.

[78] Xu, Y., Patnayakuni, R., Wang, H., 2013. Logarithmic least squares method to priority for group decision making with incomplete fuzzy preference relations. Applied Mathematical Modelling 37 (4), 2139-2152.

[79] Xu, Z. S, 2004. A method based on linguistic aggregation operators for group decision making with linguistic preference relations. Information Sciences 166 (1-4), 19-30.

[80] Xu, Z. S, 2004. On compatibility of interval fuzzy preference relations. Fuzzy Optimization and Decision Making 3 (3), 217-225.

[81] Xu, Z. S., 2004. Goal programming models for obtaining the priority vector of incomplete fuzzy preference relation. International Journal of Approximate Reasoning 36 (3), 261-270.

[82] Xu, Z. S., 2005. Interactive approach based on incomplete complementary judgement matrices to group decision making. Control and decision 20, 913-916.

[83] Xu, Z. S., 2005. A procedure for decision making based on incomplete fuzzy preference relation. Fuzzy Optimization and decision Making 4, 175-189. 
[84] Xu, Z. S., 2006. A Practical Procedure for Group Decision Making under Incomplete Multiplicative Linguistic Preference Relations. Group Decision and Negotiation. 15 (6), 581-590.

[85] Xu, Z. S., 2006. Incomplete linguistic preference relations and their fusion. Information Fusion 7 (3), 331-337.

[86] Xu, Z. S, 2007. A survey of preference relations. International Journal of General Systems $36(2), 179-203$.

[87] $\mathrm{Xu}, \mathrm{Z} . \mathrm{S}, 2007$. Intuitionistic preference relations and their application in group decision making. Information Sciences 177 (11), 2363 - 2379.

[88] Xu, Z. S., 2010. An integrated model-based interactive approach to fuzzy multiattribute group decision making with incomplete preference information. Fuzzy Optimization and Decision Making 9, 333-357.

[89] Xu, Z. S, 2010. Interactive group decision making procedure based on uncertain multiplicative linguistic preference relations. Journal of Systems Engineering and Electronics 21 (3), $408-415$.

[90] Xu, Z. S, 2012. A consensus reaching process under incomplete multiplicative preference relations. International Journal of General Systems 41 (4), 333-351.

[91] Xu, Z. S., 2014. Hesitant Fuzzy Sets Theory Series: Studies in Fuzziness and Soft Computing, Vol. 314. Springer International Publishing Switzerland.

[92] Xu, Z. S., Cai, X., 2009. Incomplete interval-valued intuitionistic fuzzy preference relations. International Journal of General Systems 38 (8), 871-886.

[93] Xu, Z. S, Cai, X., Szmidt, E., 2011. Algorithms for estimating missing elements of incomplete intuitionistic preference relations. International Journal of Intelligent Systems 26 (9), $787-813$.

[94] Xu, Z. S, Chen, J., 2008. Group decision-making procedure based on incomplete reciprocal relations. Soft Computing 12, 515-521.

[95] Xu, Z. S , Chen, J., 2008. Some models for deriving the priority weights from interval fuzzy preference relations. European Journal of Operational Research 184 (1), 266-280.

[96] Xu, Z.S., Xia, M., 2014. Iterative algorithms for improving consistency. Journal of Operational Research Society 65 (5), 708-722. 
[97] Yager, R., 1981. A new methodology for ordinal multiobjective decisions based on fuzzy sets. Decision Sciences 12, 589-600.

[98] Yang, X., Yu, D., Yang, J., Wei, L., 2009. Dominance-based rough set approach to incomplete interval-valued information system. Data \& Knowledge Engineering 68 (11), 13311347.

[99] Dong, Y., Herrera-Viedma, E., 2015. Consistency-driven automatic methodology to set interval numerical scales of 2-tuple linguistic term sets and its use in the linguistic GDM with preference relation. IEEE Transactions on Cybernetics, in press.

[100] Zadeh, L. A., 1965. Fuzzy sets. Information and Control 8 (3), 338-357.

[101] Zadeh, L. A., 1975. The concept of a linguistic variable and its application to approximate reasoning-i. Information Sciences 8, 199-249.

[102] Zhang, G., Dong, Y., Xu, Y., 2012. Linear optimization modeling of consistency issues in group decision making based on fuzzy preference relations. Expert Systems with Applications $39,2415-2420$.

[103] Zhu, B., Xu, Z. S., 2014. Consistency measures for hesitant fuzzy linguistic preference relations. IEEE Transactions on Fuzzy Systems 22 (1), 35-45. 